EPJ Web of Conferences 49, 18014 (2013)

DOI: $10.1051 /$ epjconf/20134918014

(C) Owned by the authors, published by EDP Sciences, 2013

\title{
ATLAS Triggering on SUSY in 2012
}

\author{
Anna Sfyrla ${ }^{1, a}$ On behalf of the ATLAS Collaboration. \\ ${ }^{1}$ CERN
}

\begin{abstract}
In 2012 the LHC has been operating at a higher center-of-mass energy and higher instantaneous luminosity compared to 2011 , providing the experiments with unprecedented amounts of hadron collision data. This document summarizes how the challenge of triggering on the 2012 LHC data on new physics signatures, such as supersymmetry (SUSY), was addressed by the ATLAS experiment.
\end{abstract}

\section{Introduction}

The LHC [1] operational conditions in 2012 presented a big challenge for the ATLAS trigger [2]; the higher center-of-mass energy and luminosity resulted in trigger rates an order of magnitude higher in 2012 than in 2011, as well as in large non-linearity in trigger rates as a function of the luminosity, due to the pile-up. Triggering on new physics, such as SUSY, represented a particular challenge, as trigger selections had to be inclusive enough to provide a broad coverage of phase-space. This was taken into account in the design of the 2012 trigger selection composition that was developed with the strategy to be as inclusive as possible in object thresholds and multiplicities.

\section{The ATLAS trigger system and the 2012 trigger menu}

The ATLAS trigger system consists of a hardware-based component, the Level-1 (L1), and two software parts, the Level-2 (L2) and the Event Filter (EF). The L2 and the EF are referred together as High Level Trigger (HLT). Cost reasons define the limitation in the output bandwidth of both the L1 and the HLT. The detector readout bandwidth and the processing power in the HLT limit the L1 output rate to $75 \mathrm{kHz}$ and the L2 output rate to $6 \mathrm{kHz}$. The offline computing capacity for storing and processing data promptly limits the EF output rate to $\mathrm{O}(400 \mathrm{~Hz})$. An additional $\mathrm{O}(200 \mathrm{~Hz})$ is stored for later reconstruction, in the so-called 'delayed stream'.

The trigger menu, i.e. the list of trigger selection criteria used for data taking, consists of 'primary' triggers, which are used for physics measurements and are typically running unprescaled; 'support' triggers, which are used for efficiency and performance measurements or monitoring, and are typically running at a small rate (of the order of $0.5 \mathrm{~Hz}$ each); and 'calibration' triggers, which are used for detector calibrations and are often running at high rate but storing very small events with the relevant information from the detector needed for the calibrations. The total of the selections have to respect all three-level trigger limitations, what makes the optimal distribution of available bandwidth a challenge.

ae-mail: anna.sfyrla@cern.ch

This is an Open Access article distributed under the terms of the Creative Commons Attribution License 2.0, which permits unrestricted use, distribution, and reproduction in any medium, provided the original work is properly cited. 
The distribution is driven by physics priorities, and ATLAS has chosen to give the most generic triggers the larger fraction of the bandwidth. Single electron and muon triggers typically use $50 \mathrm{~Hz}$ each; generic triggers, such as multi-jets and multi-leptons, typically use 5-15 Hz; and specialized triggers are given $\approx 1 \mathrm{~Hz}$. About $20 \%$ of the bandwidth is dedicated to the supporting triggers. The main triggers that compose the ATLAS 2012 trigger menu are shown in Table 1.

Table 1. The main ATLAS triggers in 2012. The total rate corresponds to the full menu that includes many more triggers than what is listed in this table.

\begin{tabular}{|c|c|c|c|c|c|}
\hline \multirow{2}{*}{ Trigger } & \multirow{2}{*}{ Typical offline selection } & \multicolumn{2}{|c|}{ Trigger Selection } & \multirow{2}{*}{$\begin{array}{c}\text { L1 Peak } \\
\text { Rate }(\mathbf{k H z}) \\
L_{\text {peak }}=7 \mathrm{e} 33 / \mathrm{cm}^{2} \mathrm{~s} \\
\end{array}$} & \multirow{2}{*}{$\begin{array}{c}\text { EF Avg. } \\
\text { Rate }(\mathbf{H z}) \\
L_{\text {avg. }}=5 \mathrm{e} 33 / \mathrm{cm}^{2} \mathrm{~s} \\
\end{array}$} \\
\hline & & L1 & EF & & \\
\hline \multirow{2}{*}{ Single leptons } & Single iso $\mu, p_{T}>25 \mathrm{GeV}$ & $15 \mathrm{GeV}$ & $24 \mathrm{GeV}$ & 8 & 45 \\
\hline & Single iso $e, p_{T}>25 \mathrm{GeV}$ & $18 \mathrm{GeV}$ & $25 \mathrm{GeV}$ & 17 & 70 \\
\hline \multirow{5}{*}{ Two leptons } & Two $\mu$ 's, each $p_{T}>15 \mathrm{GeV}$ & $2 \times 10 \mathrm{GeV}$ & $2 \times 13 \mathrm{GeV}$ & 1 & 5 \\
\hline & Two $\mu$ 's, $p_{T}>20,10 \mathrm{GeV}$ & $15 \mathrm{GeV}$ & $18,8 \mathrm{GeV}$ & 8 & 8 \\
\hline & Two $e$ 's, each $p_{T}>15 \mathrm{GeV}$ & $2 \times 10 \mathrm{GeV}$ & $2 \times 12 \mathrm{GeV}$ & 6 & 8 \\
\hline & Two $e$ 's, $p_{T}>25,10 \mathrm{GeV}$ & $18 \mathrm{GeV}$ & $25,7 \mathrm{GeV}$ & 17 & 5 \\
\hline & Two $\tau$ 's, $p_{T}>45,30 \mathrm{GeV}$ & $15,11 \mathrm{GeV}$ & $29,20 \mathrm{GeV}$ & 12 & 12 \\
\hline \multirow{2}{*}{ Two photons } & "Two $\gamma$ 's, each $p_{T}>25 \mathrm{GeV}$ & $2 \times 10 \mathrm{GeV}$ & $2 \times 20 \mathrm{GeV}$ & 6 & 10 \\
\hline & Two $\gamma$ 's, $p_{T}>40,30 \mathrm{GeV}$ & $16,12 \mathrm{GeV}$ & $35,25 \mathrm{GeV}$ & 6 & 7 \\
\hline \multirow{2}{*}{ Single jet } & Jet $(R=0.4), p_{T}>360 \mathrm{GeV}$ & \multirow{2}{*}{$75 \mathrm{GeV}$} & $370 \mathrm{GeV}$ & \multirow{2}{*}{2} & 5 \\
\hline & Jet $(R=1.0), p_{T}>470 \mathrm{GeV}$ & & $460 \mathrm{GeV}$ & & 2 \\
\hline$E_{T}^{\text {miss }}$ & $E_{T}^{m i s s}>150 \mathrm{GeV}$ & $40 \mathrm{GeV}$ & $80 \mathrm{GeV}$ & 2 & 17 \\
\hline \multirow{3}{*}{ Multi-jets } & 4 jets, each $p_{T}>85 \mathrm{GeV}$ & \multirow{3}{*}{$4 \times 15 \mathrm{GeV}$} & $4 \times 80 \mathrm{GeV}$ & \multirow{3}{*}{1} & 8 \\
\hline & 5 jets, each $p_{T}>60 \mathrm{GeV}$ & & $5 \times 55 \mathrm{GeV}$ & & 2 \\
\hline & 6 jets, each $p_{T}>50 \mathrm{GeV}$ & & $6 \times 45 \mathrm{GeV}$ & & 4 \\
\hline$b$-jets & $\begin{array}{l}4 \text { jets, each } p_{T}>50 \mathrm{GeV} \\
\text { out of which one is } b \text {-tagged }\end{array}$ & $4 \times 15 \mathrm{GeV}$ & $\begin{array}{l}4 \times 45 \mathrm{GeV} \\
\text { plus } b-\text { tag }\end{array}$ & 1 & 4 \\
\hline \multicolumn{4}{|l|}{ Total } & $<75$ & 400 \\
\hline
\end{tabular}

\section{SUSY triggers}

The triggers outlined in Table 1 are extensively used by SUSY searches, and in some cases, their thresholds were adjusted to fit the SUSY requirements (e.g. multi-jet triggers). Several additional selections were added to the ATLAS trigger menu, to extend the trigger coverage for SUSY searches. Some examples are outlined in Table 2. Additionally, the SUSY searches motivated the introduction in the delayed stream of looser hadronic and $E_{\mathrm{T}}^{\text {miss }}$ triggers, compared to what is in the prompt stream. Examples of such triggers are given in Table 3.

\section{Trigger performance improvements in 2012}

To cope with the increased energy, luminosity and pile-up conditions of the 2012 data taking, the ATLAS experiment deployed improvements in the trigger selections and algorithms. The improvements mostly affecting SUSY selections were implemented in jet and missing transverse momentum $\left(E_{\mathrm{T}}^{\mathrm{miss}}\right)$ triggers. A summary of these improvements can be found elsewhere [3]. 
Table 2. SUSY-motivated triggers in the 2012 trigger menu. The $\Delta \phi$ selection is applied at EF, between the $E_{\mathrm{T}}^{\text {miss }}$ and the two leading jets with $E_{T}>45 \mathrm{GeV} . \mathrm{H}_{\mathrm{T}}$ is defined as the sum of jets with $E_{T}>45 \mathrm{GeV}$, and is calculated in events that already satisfied the requirement of a leading jet $E_{T}>145 \mathrm{GeV}$. In some combined triggers, EF-only selections are implemented for jets and $E_{\mathrm{T}}^{\text {miss }}$ when the $\mathrm{L} 2$ rejection is sufficient; this feature provides optimal online to offline correlations, as at $\mathrm{EF}$ the jet and $E_{\mathrm{T}}^{\text {miss }}$ reconstruction is similar to offline.

\begin{tabular}{|c|c|c|}
\hline Selection & EF trigger election & $\begin{array}{c}\text { EF Avg. Rate } \\
(\mathbf{H z}) \\
L_{\text {avrg }}=5 \mathrm{e} 33 / \mathrm{cm}^{2} \mathrm{~s} \\
\end{array}$ \\
\hline $\begin{array}{l}\text { Single jet } \\
\& E_{\mathrm{T}}^{\text {miss }}\end{array}$ & $\begin{array}{c}\text { Jet } E_{\mathrm{T}}>145 \mathrm{GeV} \\
\text { \& EF-only } E_{\mathrm{T}}^{\text {miss }}>70 \mathrm{GeV}\end{array}$ & 8 \\
\hline $\begin{array}{c}\text { Single jet } \\
\& E_{\mathrm{T}}^{\mathrm{miss}} \& \Delta \phi\left(\text { jet }, E_{\mathrm{T}}^{\mathrm{miss}}\right)\end{array}$ & $\begin{array}{c}\text { Jet } E_{\mathrm{T}}>80 \mathrm{GeV} \\
\& E_{\mathrm{T}}^{\text {miss }}>70 \mathrm{GeV} \& \Delta \phi>1.0 \mathrm{rad}\end{array}$ & 8 \\
\hline $\mathrm{H}_{\mathrm{T}}$ & $>700 \mathrm{GeV}$ & 8 \\
\hline $\begin{array}{l}\text { Single electron } \\
\quad \& E_{\mathrm{T}}^{\text {miss }}\end{array}$ & $\begin{array}{c}\text { Electron } p_{\mathrm{T}}>25 \mathrm{GeV} \\
\& \text { EF-only } E_{\mathrm{T}}^{\text {miss }}>35 \mathrm{GeV}\end{array}$ & 26 \\
\hline $\begin{array}{c}\text { Single muon } \\
\& \text { single jet \& } E_{\mathrm{T}}^{\text {miss }}\end{array}$ & $\begin{array}{c}\text { Muon } p_{\mathrm{T}}>24 \mathrm{GeV} \\
\& \text { jet } E_{\mathrm{T}}>65 \mathrm{GeV} \& \text { EF-only } E_{\mathrm{T}}^{\text {miss }}>40 \mathrm{GeV}\end{array}$ & 15 \\
\hline $\begin{array}{l}\text { Single photon } \\
\& E_{\mathrm{T}}^{\text {miss }} \\
\end{array}$ & $\begin{array}{c}\text { Photon } p_{\mathrm{T}}>40 \mathrm{GeV} \\
\& \text { EF-only } E_{\mathrm{T}}^{\text {miss }}>60 \mathrm{GeV}\end{array}$ & 5 \\
\hline 3 electrons & $p_{\mathrm{T}}>18,2 \times 7 \mathrm{GeV}$ & $<1$ \\
\hline 3 muons & $p_{\mathrm{T}}>18,2 \times 4 \mathrm{GeV}$ & $<1$ \\
\hline \multirow{2}{*}{3 electrons \& muons } & $p_{\mathrm{T}}>2 \times 7(e), 6(\mu) \mathrm{GeV}$ & $<1$ \\
\hline & $p_{\mathrm{T}}>7(e), 2 \times 6(\mu) \mathrm{GeV}$ & $<1$ \\
\hline
\end{tabular}

Table 3. Triggers in the delayed stream, introduced to enhance the trigger coverage for searches for which SUSY was one of the main motivations. The $E_{\mathrm{T}}^{\text {miss }}$ selection differs not only at the HLT but also at the L1, where it is looser by $5 \mathrm{GeV}$. The jet variable $R$ corresponds to the jet cone size.

\begin{tabular}{|l|c|c|}
\hline \multirow{2}{*}{ Trigger } & \multicolumn{2}{|c|}{ EF trigger Selection } \\
\cline { 2 - 3 } & Prompt Stream & Delayed Stream \\
\hline \hline \multirow{3}{*}{ Multi-jets } & $4 \times 80 \mathrm{GeV}$ & $4 \times 65 \mathrm{GeV}$ \\
\cline { 2 - 3 } & $5 \times 55 \mathrm{GeV}$ & $5 \times 45 \mathrm{GeV}$ \\
\cline { 2 - 3 } & $6 \times 45 \mathrm{GeV}$ & \\
\hline \hline $\mathrm{H}_{\mathrm{T}}$ & $700 \mathrm{GeV}$ & $500 \mathrm{GeV}$ \\
\hline \hline Single jet $(R=1.0)$ & $460 \mathrm{GeV}$ & $360 \mathrm{GeV}$ \\
\hline \hline$E_{\mathrm{T}}^{\text {miss }}$ & $80 \mathrm{GeV}$ & $60 \mathrm{GeV}$ \\
\hline
\end{tabular}

\section{Summary}

The improvements made to jet and $E_{\mathrm{T}}^{\text {miss }}$ triggers for 2012 together with new trigger selections and the addition of a delayed processing stream have allowed ATLAS to meet the challenges of increased luminosity and pile-up and maintain excellent efficiency for SUSY signals in 2012 data taking.

\section{References}

[1] L. Evans and P. Bryant (editors) 2008 JINST 3 S08001.

[2] ATLAS Collaboration, 2008 JINST 3 S08003.

[3] ATLAS Collaboration, ATL-DAQ-PUB-2012-002, https://cds.cern.ch/record/1492192. 